\title{
Magneto-thermal evolution of neutron stars
}

\author{
J. A. Pons ${ }^{1}$, J. A. Miralles ${ }^{1}$, and U. Geppert ${ }^{2}$ \\ 1 Departament de Física Aplicada, Universitat d'Alacant, Ap. Correus 99, 03080 Alacant, Spain \\ e-mail: jose.pons@ua.es \\ ${ }^{2}$ German Aerospace Center, Institute for Space Systems, Rutherfordstr. 2, 12489 Berlin, Germany
}

Received 27 October 2008 / Accepted 14 December 2008

\section{ABSTRACT}

\begin{abstract}
Context. The presence of magnetic fields in the crust of neutron stars (NSs) causes a non-spherically symmetric temperature distribution. The strong temperature dependence of the magnetic diffusivity and thermal conductivity, together with the heat generated by magnetic dissipation, couple the magnetic and thermal evolution of NSs, which can no longer be formulated as separated onedimensional problems.

Aims. We study the mutual influence of thermal and magnetic evolution in a neutron star's crust in axial symmetry. Taking realistic microphysical inputs into account, we find the heat released by Joule effect consistent with the circulation of currents in the crust, and we incorporate its effects in 2D cooling calculations.

Methods. We solve the induction equation numerically using a hybrid method (spectral in angles, but a finite-differences scheme in the radial direction), coupled to the thermal diffusion equation. To improve the boundary conditions, we also revisit the envelope stationary solutions updating the well known $T_{\mathrm{b}}-T_{\mathrm{s}}$-relations to include the effect of $2 \mathrm{D}$ heat transfer calculations and new microphysical inputs.

Results. We present the first longterm 2D simulations of the coupled magneto-thermal evolution of neutron stars. This substantially improves previous works in which a very crude approximation in at least one of the parts (thermal or magnetic diffusion) has been adopted. Our results show that the feedback between Joule heating and magnetic diffusion is strong, resulting in a faster dissipation of the stronger fields during the first $10^{5}-10^{6}$ years of an NS's life. As a consequence, all neutron stars born with fields over a critical value $\left(>5 \times 10^{13} \mathrm{G}\right)$ reach similar field strengths $\left(\approx 2-3 \times 10^{13} \mathrm{G}\right)$ at late times. Irrespective of the initial magnetic field strength, the temperature becomes so low after $10^{6}$ years that the magnetic diffusion timescale becomes longer than the typical ages of radiopulsars, thus apparently resulting in no dissipation of the field in old NS. We also confirm the strong correlation between the magnetic field and the surface temperature of relatively young NSs discussed in preliminary works. The effective temperature of models with strong internal toroidal components are systematically higher than those of models with purely poloidal fields, due to the additional energy reservoir stored in the toroidal field that is gradually released as the field dissipates.
\end{abstract}

Key words. stars: neutron - stars: evolution - stars: magnetic fields

\section{Introduction}

The neutron star (NS) magnetic field (MF) maintained by electric currents circulating in the crust modifies the crustal temperature distribution by means of two mechanisms. The first one stems from the anisotropy of thermal conductivity in presence of a strong MF and causes important changes in how heat flux flows from the core through the crust and envelope up to the surface. The second mechanism is the generation of heat caused by MF dissipation that results in a non-spherically symmetric allocation of heating sources. Therefore, besides its tensorial character due to the presence of the MF (which eventually results in the Hall drift of the field), the magnetic diffusivity becomes a non-spherically symmetric quantity. Under these conditions, an initially purely dipolar field evolves very quickly in a magnetized NS to generate a complex structure including toroidal fields and/or higher order multipoles. Different studies of magnetohydrostatic equilibrium configurations indicate that stable configurations require both toroidal and poloidal components (see e.g. Reisenegger 2008, and references therein).

The complexity of the problem has limited previous works to partial studies of the complete problem. The anisotropic heat flux and its consequences for the - in principle observable surface temperature $\left(T_{\mathrm{s}}\right)$ distribution has been considered by
Geppert et al. (2004, 2006) and Pérez-Azorín et al. (2006a,b), but for fixed, prescribed MF. The longterm effect of Joule heating has also been recently discussed by Pons et al. (2007); Aguilera et al. (2008c) or Urpin \& Konenkov (2008) (see also references therein), although in a very crude approach. In Aguilera et al. (2008b) a full 2D cooling code is used to describe the temperature evolution, but the Joule heating rate is estimated by an analytical approximation and considered to be uniform. In Urpin \& Konenkov (2008) the former results of Miralles et al. (1998) are revisited in the context of high field radio-pulsars, but with the simplified approach of considering the diffusion of a one-mode (dipolar) poloidal field, without actually performing consistent simulations, including the temperature evolution. On the other hand, the evolution of the MF including the influence of the Hall term at early times has been studied in Pons \& Geppert (2007). The Hall drift causes a somewhat faster dissipation for strong initial fields due to the reorganization of the field on smaller scales. In this work the temporal evolution of the temperature has been prescribed according to a generally accepted cooling law and assumed to be the same in the whole crust. The effect of ambipolar diffusion in the liquid core can also be relevant, as recently studied for example in Hoyos et al. (2008).

One of the causes of complex MF geometries in the crust is the non-spherically symmetric magnetic diffusivity. It is 
effective even if the Hall drift is negligible. Since the temperature within the crust is no longer uniform, the magnetic diffusivity in this approach becomes a function of both the radial and the polar coordinates, thus rendering the one-mode approximation inappropriate. Up to now, the effect of the dependence of the magnetic diffusivity on the polar angle $\theta$ (if axial symmetry is assumed) has not been considered. In some studies about cooling of NSs, the source term in the thermal diffusion equation includes an angular-averaged Joule heating rate, where the heat production is uniform in spherical shells at a given radius $r$ (Page et al. 2000; Aguilera et al. 2008c; Urpin \& Konenkov 2008). These are not fully consistent calculations, because the currents can be very intense locally and release heat in a highly non-spherically symmetric way. Therefore, it is important to attack the problem of the full magneto-thermal evolution of NSs by solving simultaneously the induction equation and the heat transfer equation, taking into account the anisotropy of the thermal conductivity and the electrical resistivity tensors in a consistent way. This is the main goal of this paper, in which we solve this problem (in axial symmetry) for the first time. The aim is to provide a self-consistent model for the coupled evolution of the MF and temperature in axially symmetric NSs, in general relativity, and including the effect of angular variations of temperature in the magnetic diffusivity and the thermal conductivity. We also revisit the envelope stationary solutions, while updating the well known $T_{\mathrm{b}}-T_{\mathrm{s}}$-relations to include the effect of $2 \mathrm{D}$ heat transfer calculations and new advances in microphysical ingredients (phonons, ion-ion interactions). We provide new fits to solutions of the equilibrium magnetic envelope that can be used as boundary conditions in multidimensional cooling simulations.

The paper is organized as follows. In the next section we derive and present the basic equations together with the boundary conditions. Next we describe the input microphysics and discuss the effect of superfluid neutrons on the heat flux anisotropy. The next section is devoted to presenting the magneto-thermal evolution. Finally we discuss the results by comparing them with observable consequences.

\section{Basic equations}

The thermal evolution of the NS is calculated using the code described in Aguilera et al. (2008b). For the evolution of the MF we basically follow the formalism already used in Pons \& Geppert (2007). We address the interested reader to these references for more details about the cooling code and the MF evolution code that we have merged and coupled to perform the simulations presented later in this paper. In the next sections we just summarize the basic equations that are solved with the purpose of introducing notation and for the sake of completeness.

\subsection{Heat transfer equation}

Assuming that deformations with respect to the spherically symmetric case due to rotation, MF, and temperature distribution do not affect the metric in the interior of an NS, we use the standard static metric

$\mathrm{d} s^{2}=-c^{2} \mathrm{e}^{2 v(r)} \mathrm{d} t^{2}+\mathrm{e}^{2 \lambda(r)} \mathrm{d} r^{2}+r^{2} \mathrm{~d} \Omega^{2}$,

where $v(r)$ is the relativistic redshift correction and $\lambda(r)$ the length correction factor, $\mathrm{e}^{-2 \lambda(r)}=1-2 G M(r) / c^{2} r$. Using this background metric, the thermal evolution of an NS can be described by the energy balance equation

$c_{\mathrm{v}} \mathrm{e}^{v} \frac{\partial T}{\partial t}+\boldsymbol{\nabla} \cdot\left(\mathrm{e}^{2 v} \boldsymbol{F}\right)=\mathrm{e}^{2 v}\left(-Q_{v}+Q_{\mathrm{h}}\right)$ where $c_{\mathrm{v}}$ is the specific heat per unit volume and $Q_{v}$ the energy loss by neutrino emission while $Q_{\mathrm{h}}$ stands for the Joule heating rate. In this study we neglect other heating mechanisms than Joule heating, such as accretion or frictional heating at the core crust interface. In the diffusion limit, the heat flux is simply

$\boldsymbol{F}=-\mathrm{e}^{-v} \hat{\kappa} \cdot \nabla\left(\mathrm{e}^{v} T\right)$

where $\hat{\kappa}$ is the thermal conductivity tensor that depends on both coordinates $(r, \theta)$ through its dependence on temperature and MF. The differential operator $\nabla$ associated to the spatial metric $\mathrm{e}^{2 \lambda(r)} \mathrm{d} r^{2}+r^{2} \mathrm{~d} \Omega^{2}$ must include the corresponding metric factors (i.e. $\mathrm{e}^{-\lambda(r)} \frac{\partial}{\partial r}$ for the radial component). An explicit representation in terms of the metric scale factors can be found in Rädler et al. (2001). We solve Eqs. (2) and (3) numerically following the same scheme as described in Geppert et al. (2004) and Pérez-Azorín et al. (2006a).

\subsection{The induction equation}

The Maxwell equations (in the Gaussian system) relative to an observer at rest (Eulerian observer) for the static metric given by Eq. (1) are

$\nabla \cdot \boldsymbol{E}=4 \pi \rho_{\mathrm{e}}$

$\frac{1}{c} \frac{\partial \boldsymbol{E}}{\partial t}=\boldsymbol{\nabla} \times\left(\mathrm{e}^{v} \boldsymbol{B}\right)-\frac{4 \pi}{c} \mathrm{e}^{v} \boldsymbol{j}$

$\boldsymbol{\nabla} \cdot \boldsymbol{B}=0$

$\frac{1}{c} \frac{\partial \boldsymbol{B}}{\partial t}=-\boldsymbol{\nabla} \times\left(\mathrm{e}^{v} \boldsymbol{E}\right)$,

where again the differential operators are defined according to the spatial coordinate system, $\boldsymbol{E}$ and $\boldsymbol{B}$ are the electric and magnetic field measured by the Eulerian observer, as well as $\rho_{\mathrm{e}}$ and $j$ that represent the electric charge and current density, respectively.

In the comoving frame, which corresponds to the Eulerian frame, since matter is at rest, electric field and density current are related through Ohm's law. This law establishes a proportionality between the current and the electric field, which can be written as $\boldsymbol{j}=\sigma \boldsymbol{E}, \sigma$ being the electric conductivity. In the presence of strong MFs, $\sigma$ becomes a tensor and Ohm's law must be written as $\boldsymbol{j}=\hat{\sigma} \boldsymbol{E}$ or equivalently $\boldsymbol{E}=\hat{\mathcal{R}} \boldsymbol{j}$, where $\hat{\mathcal{R}} \equiv \hat{\sigma}^{-1}$ is the resistivity tensor. This tensor can be decomposed in symmetric and antisymmetric parts (Landau \& Lifshitz 1960). The symmetric part, for nonquantizing fields is isotropic and determined by the scalar $\frac{1}{\sigma_{\|}}$, where $\sigma_{\|}$is the electric conductivity in the direction of the MF. The antisymmetric part of the resistivity tensor can be represented by a vector proportional to $\boldsymbol{B}$. The electric field $\boldsymbol{E}$ is then written in the form (Ziman 1979)

$\boldsymbol{E}=\frac{1}{\sigma_{\|}} \boldsymbol{j}+\frac{1}{e n_{\mathrm{e}} c} \boldsymbol{B} \times \boldsymbol{j}$

where $n_{\mathrm{e}}$ is the electron number density and $e$ the elementary charge.

If we neglect the displacement current term in the AmpèreMaxwell equation (second equation in 4), and make use of Eq. (5), the Faraday induction equation (fourth equation in 4) can be written as

$\frac{\partial \boldsymbol{B}}{\partial t}=-\boldsymbol{\nabla} \times\left\{\eta \boldsymbol{\nabla} \times\left(\mathrm{e}^{v} \boldsymbol{B}\right)+\frac{c}{4 \pi e n_{\mathrm{e}}}\left[\boldsymbol{\nabla} \times\left(\mathrm{e}^{v} \boldsymbol{B}\right)\right] \times \boldsymbol{B}\right\}$

where we have introduced the magnetic diffusivity $\eta \equiv \frac{c^{2}}{4 \pi \sigma_{\|}}$. 
There are two main differences from the induction equation employed in Pons \& Geppert (2007): the relativistic factors are included and the magnetic diffusivity is not assumed to be spherically symmetric.

Since both $n_{\mathrm{e}}$ and the metric factor $\mathrm{e}^{v}$ only depend on the radial coordinate (it is justified to neglect the structural deformations induced by MFs), the nonlinear Hall term can be treated in the same way as done by Pons \& Geppert (2007). However, the magnetic diffusivity through its temperature dependence also depends on the polar angle, $\eta=\eta(r, \theta)$, thus requiring an extension of the previous formalism. The influence of the Hall term at early times has been studied in Pons \& Geppert (2007), resulting in a somewhat faster dissipation for strong initial fields due to the reorganization of the field on smaller scales. The numerical treatment of the non-linear term is complex and very computationally-limited. In this paper our goal is to perform coupled (magneto-thermal) longterm simulations, thus from now on we focus on the linear part of the induction equation.

The linear part of the induction Eq. (6) reads

$\frac{\partial \boldsymbol{B}}{\partial t}=-\nabla \times\left[\eta \nabla \times\left(\mathrm{e}^{v} \boldsymbol{B}\right)\right]$.

We decompose the MF into its poloidal and toroidal part (Rädler et al. 2001)

$\boldsymbol{B}=\boldsymbol{B}_{\mathrm{pol}}+\boldsymbol{B}_{\text {tor }}$,

and use their representation in terms of two scalar functions $\Phi(r, \theta)$ and $\Psi(r, \theta)$ :

$\boldsymbol{B}_{\mathrm{pol}}=-\boldsymbol{\nabla} \times(\boldsymbol{r} \times \boldsymbol{\nabla} \Phi), \quad \boldsymbol{B}_{\mathrm{tor}}=-\boldsymbol{r} \times \boldsymbol{\nabla} \Psi$.

We now expand the functions $\Phi, \Psi$, and $\eta$ in a series of spherical harmonics (in the axisymmetric case) as follows:

$\Phi=\frac{1}{r} \sum_{n=1}^{\infty} \Phi_{n}(r, t) Y_{n}(\theta)$,

$\Psi=\frac{1}{r} \sum_{n=1}^{\infty} \Psi_{n}(r, t) Y_{n}(\theta)$,

$\eta=\sum_{n=0}^{\infty} \eta_{n}(r, t) Y_{n}(\theta)$,

where $Y_{n}$ is the spherical harmonic $Y_{n}^{m}$ for $m=0$.

The poloidal and toroidal parts of the MF can be written as

$$
\begin{aligned}
\boldsymbol{B}_{\mathrm{pol}} & =\frac{1}{r^{2}} \sum_{n} n(n+1) \Phi_{n} Y_{n} \boldsymbol{e}_{r}+\frac{\mathrm{e}^{-\lambda}}{r} \sum_{n} \frac{\partial \Phi_{n}}{\partial r} \frac{\mathrm{d} Y_{n}}{\mathrm{~d} \theta} \boldsymbol{e}_{\theta}, \\
\boldsymbol{B}_{\mathrm{tor}} & =-\left(\frac{1}{r} \sum_{n} \Psi_{n} \frac{\mathrm{d} Y_{n}}{\mathrm{~d} \theta}\right) \boldsymbol{e}_{\phi},
\end{aligned}
$$

where $\boldsymbol{e}_{r}, \boldsymbol{e}_{\theta}, \boldsymbol{e}_{\phi}$ are unitary vectors associated to the spatial metric. Inserting this expression into Eq. (7) and using the quantities $I_{k^{\prime} k}^{n}$ and $I^{(1)}$ defined in Geppert \& Wiebicke (1991, Eqs. (59) and (60)) which are related to the Clebsch-Gordan coefficients, we arrive at the following evolution equations for the poloidal and toroidal scalar functions:

$$
\begin{aligned}
\frac{\partial \Phi_{n}}{\partial t}= & \mathrm{e}^{v} \sum_{k, k^{\prime}} \eta_{k^{\prime}}\left(I_{k^{\prime} k}^{n}-I^{(1)}\right) \\
& \times\left[\mathrm{e}^{-2 \lambda} \frac{\partial^{2} \Phi_{k}}{\partial r^{2}}+\mathrm{e}^{-2 \lambda}\left(\frac{\mathrm{d} v}{\mathrm{~d} r}-\frac{\mathrm{d} \lambda}{\mathrm{d} r}\right) \frac{\partial \Phi_{k}}{\partial r}-\frac{k(k+1)}{r^{2}} \Phi_{k}\right]
\end{aligned}
$$

and

$$
\begin{aligned}
\frac{\partial \Psi_{n}}{\partial t}= & \sum_{k, k^{\prime}}\left\{\left(I_{k^{\prime} k}^{n}-I^{(1)}\right) \mathrm{e}^{-\lambda} \frac{\partial}{\partial r}\left(\eta_{k^{\prime}} \mathrm{e}^{-\lambda} \frac{\partial\left(\mathrm{e}^{v} \Psi_{k}\right)}{\partial r}\right)\right. \\
& \left.-\eta_{k^{\prime}} I_{k^{\prime} k}^{n} \frac{k(k+1)}{r^{2}} \mathrm{e}^{v} \Psi_{k}\right\} .
\end{aligned}
$$

If $\eta=\eta(r, t)$ only $k^{\prime}=0$ contributes and, taking the nonrelativistic limit $\left(\mathrm{e}^{v}=\mathrm{e}^{\lambda}=1\right)$, the purely diffusive Eqs. (8) of Pons \& Geppert (2007) with $D_{n m}=C_{n m}=0$ are recovered. No coupling between poloidal and toroidal component appears, since this coupling can only be obtained by including the Hall drift term.

\subsection{Microphysics}

The microphysical ingredients that enter into the heat transport and induction equation are the specific heat, the thermal conductivity, the neutrino emissivity, and the magnetic diffusivity. In the solid crust, the dominant contribution to the specific heat is that from electrons and ions, while electrons, lattice phonons, and collective modes of superfluid neutrons contribute to the thermal conductivity. We refer the reader to a detailed description of the employed equations of state, the thermal conductivity, the specific heat, and the neutrino emissivities, given in Sect. 4 of Aguilera et al. (2008b). In this paper we assume the minimal cooling scenario (Page et al. 2004) which includes neutrino emission from the Cooper pair breaking and formation process, but we do not take any direct Urca process into account, due either to nucleons or to exotic matter (hyperons, Bose condensates, or deconfined quarks).

In addition, it has been shown by Chugunov \& Haensel (2007) that the ionic contribution to the total thermal conductivity is negligible in the crust, but it may play a role for low temperatures in the envelope. Very recently (Aguilera et al. 2008a) have studied the possible effects of collective modes of superfluid neutrons. They found that this process may dominate the thermal conductivity in the inner crust when its temperature is $\lesssim 10^{7} \mathrm{~K}$. For such relatively old NSs, heat transport by superfluid neutrons counteracts the anisotropy in the electron conductivity caused by a strong crustal field and, eventually, turns the inner crust isothermal. In this work we have updated our microphysics and also included these two new contributions to the thermal conductivity.

The only relevant contributions to the electrical resistivity are electron-phonon and electron-impurity collisions (Flowers $\&$ Itoh 1976). While the efficiency of electron-phonon collisions strongly depends on the crustal temperature, the electronimpurity scattering is much less sensitive to it. However, both processes may be strongly affected by the presence of a strong MF that suppresses the conductivity components perpendicular to the field lines (Canuto \& Chiuderi 1970; Itoh 1975). As in Pons \& Geppert (2007), we calculated the electrical resistivity by using the electron relaxation time provided by Potekhin's public code $^{1}$. We used an impurity concentration parameter of 0.1 . The definition of this parameter and a discussion about how it affects electronic transport can be found in 5.1.1 of Pérez-Azorín et al. (2006a). Jones (1999) has shown that disorder in the inner crust could result in an impurity parameter $\gtrsim 10$, which leads to higher electrical resistivity and enhanced ohmic decay. However, this effect only becomes important for sufficiently cool NSs.

\footnotetext{
${ }^{1}$ www.ioffe.rssi.ru/astro/conduct/condmag.html
} 


\subsection{Joule heating}

Joule heating couples the thermal and magnetic evolution by contributing to the source term $Q_{\mathrm{h}}$ in Eq. (2), which for large fields can result in a higher temperature and therefore a greater magnetic diffusivity. This feedback may lead to a faster dissipation of the MF if it is strong enough to really alter the temperature of the crust. In this paper, Joule heating is consistently taken into account in a cooling simulation for the first time. As the MF evolves in time, we compute the local values of the electrical current density at each point of the computational grid, which is simply

$\boldsymbol{j}(r, \theta, t)=\mathrm{e}^{-v} \frac{c}{4 \pi} \nabla \times\left(\mathrm{e}^{v} \boldsymbol{B}\right)$.

The corresponding components of the current density are

$$
\begin{aligned}
& \frac{4 \pi}{c} \boldsymbol{j}=\frac{1}{r^{2}} \sum_{n} n(n+1) \Psi_{n} Y_{n} \boldsymbol{e}_{r} \\
& +\frac{\mathrm{e}^{-\lambda-\gamma}}{r} \sum_{n} \frac{\partial\left(\mathrm{e}^{r} \Psi_{n}\right)}{\partial r} \frac{\mathrm{d} Y_{n}}{\mathrm{~d} \theta} \boldsymbol{e}_{\theta} \\
& +\frac{1}{r} \sum_{n}\left[\mathrm{e}^{-2 \lambda} \frac{\partial^{2} \Phi_{n}}{\partial r^{2}}+\frac{2 G M(r)}{c^{2} r^{2}} \frac{\partial \Phi_{n}}{\partial r}-\frac{n(n+1)}{r^{2}} \Phi_{n}\right] \frac{\mathrm{d} Y_{n}}{\mathrm{~d} \theta} \boldsymbol{e}_{\phi} .
\end{aligned}
$$

We evaluate the heating source term by

$$
Q_{\mathrm{h}}=\frac{\boldsymbol{j}^{2}}{\sigma_{\|}}
$$

where $Q_{\mathrm{h}}$ is the energy per unit time and unit volume measured by the Eulerian observer. The magnetic energy density balance equation is

$$
\frac{\partial}{\partial t}\left(\mathrm{e}^{v} \frac{B^{2}}{8 \pi}\right)=-\mathrm{e}^{2 v} \frac{\boldsymbol{j}^{2}}{\sigma_{\|}}-\nabla \cdot\left[\mathrm{e}^{2 v} \frac{1}{4 \pi} \boldsymbol{E} \times \boldsymbol{B}\right],
$$

which is easily interpreted. If we integrate over the whole volume, the magnetic energy losses are caused by Joule heating and Poynting flux through the boundaries. This latter term vanishes since we do not consider the possibility of having electromagnetic waves (i.e. we neglect displacement currents).

\subsection{Magnetic boundary conditions}

Since we restrict ourselves to MF configurations confined to the crust, the inner boundary conditions are determined by the requirement that the normal component of the MF and the tangential components of the electric field has to vanish at $r=R_{\mathrm{c}}$. This is a consequence of the assumption that the core is in a superconducting state and the Meissner-Ochsenfeld effect prevents the MF from penetrating. Therefore we apply the following boundary conditions at $r=R_{\mathrm{c}}$ :

$$
\begin{aligned}
\Phi_{n} & =0 \\
\mathrm{e}^{-\lambda} \frac{\partial \Psi_{n}}{\partial r} & =-\frac{\omega_{B} \tau}{r^{2}} \sum_{k, k^{\prime}} I^{(2)} \Psi_{k} \Psi_{k^{\prime}} .
\end{aligned}
$$

A detailed derivation is given in Pons \& Geppert (2007). In the limit of vanishing Hall-drift this reduces to $\frac{\partial \Psi_{n}}{\partial r}=0$.

For the outer $\mathrm{BC}$ we require all components of the MF to be continuous across $r=R_{\mathrm{NS}}$ to match the relativistic vacuum solution. Hence, let us first consider the stationary solution for outer space. In the absence of external currents, the toroidal component of the MF must vanish, and each multipole of the poloidal field must satisfy (in the stationary case)

$(1-z) \frac{\partial^{2} \Phi_{n}}{\partial r^{2}}+\frac{z}{r} \frac{\partial \Phi_{n}}{\partial r}-\frac{n(n+1)}{r^{2}} \Phi_{k}=0$

where $z=2 G M / c^{2} r$, which corresponds to the compactness parameter at $r=R_{\mathrm{NS}}$.

This second-order differential equation has analytical solutions for each value of $n$, although they cannot be written in a closed analytic form valid for any $n$. For example, for $n=1$ and $n=2$ we have

$\Phi_{1}=C_{1} r^{2}\left[\ln (1-z)+z+\frac{z^{2}}{2}\right]+C_{2} r^{2}$

$\Phi_{2}=C_{1} r^{3}\left[(4-3 z) \ln (1-z)+4 z-z^{2}-\frac{z^{3}}{6}\right]+C_{2} r^{3}(4-3 z)$

where $C_{1}$ and $C_{2}$ are arbitrary integration constants that must be fixed according to the value of the magnetic multipole moments. Regularity of the external solution at $r=\infty$ requires $C_{2}=0$. In general, the family of solutions of Eq. (20) for any value of $n$ can also be expressed in terms of generalized hypergeometric functions $(F([],[], z))$, also known as Barnes's extended hypergeometric functions, as follows:

$$
\begin{aligned}
\Phi_{n}= & C_{1} r^{-n} F([n, n+2],[2+2 n], z) \\
& +C_{2} r^{n+1} F([1-n,-1-n],[-2 n], z) .
\end{aligned}
$$

Note that regularity at $r=\infty$ again requires $C_{2}=0$.

For practical reasons, we write the outer boundary conditions at $r=R_{\mathrm{NS}}$ as

$$
\begin{aligned}
\frac{\partial \Phi_{n}}{\partial r} & =-\frac{n}{R_{\mathrm{NS}}} f_{n} \Phi_{n} \\
\Psi_{n} & =0
\end{aligned}
$$

where $f_{n}$ is a relativistic factor that only depends on the compactness ratio $z\left(R_{\mathrm{NS}}\right)$ (in the Newtonian limit $f_{n}=1$ ) and can be evaluated numerically or with the help of any algebraic manipulator using the form given in Eq. (23). Rädler et al. (2001) use an alternative form based on the expansion of the vacuum solutions in a series of powers of $1 / r$.

\section{Thermal boundary conditions: blanketing envelopes revisited}

The very different thermal relaxation timescales of the envelope and the crust of NSs makes any attempt to perform cooling simulations in a numerical grid that includes both regions simultaneously computationally expensive. Since radiative equilibrium is established in the low-density region much faster than the crust evolves, the usual approach is to use results of stationary, planeparallel, envelope models to obtain a phenomenological fit that relates the temperature at the bottom of the envelope $T_{\mathrm{b}}$, with the surface temperature $T_{\mathrm{s}}$. This $T_{\mathrm{s}}=T_{\mathrm{s}}\left(T_{\mathrm{b}}\right)$ phenomenological function is used to implement boundary conditions, because it allows the calculation of the surface flux for a given temperature at the base of the envelope. The location of $T_{\mathrm{b}}$ is generally chosen to correspond to some density between the neutron drip point $\rho \approx 3 \times 10^{11} \mathrm{~g} \mathrm{~cm}^{-3}$ and $\rho=10^{10} \mathrm{~g} \mathrm{~cm}^{-3}$. Examples of such models of magnetized envelopes have been constructed by Potekhin \& Yakovlev (2001) and later upgraded in Potekhin et al. (2007) 
to include the effect of the neutrino emissivity in the outer crust. They derived an analytic form of the $T_{\mathrm{b}}-T_{\mathrm{s}}$ relation that reads

$T_{\mathrm{s}}\left(B, \varphi, g, T_{\mathrm{b}}\right) \approx T_{\mathrm{s}}^{(0)}\left(g, T_{\mathrm{b}}\right) \mathcal{X}\left(B, \varphi, T_{\mathrm{b}}\right)$,

where

$T_{\mathrm{s}}^{(0)} \approx 10^{6} g_{14}^{1 / 4}\left[(7 \zeta)^{2.25}+(\zeta / 3)^{1.25}\right]^{1 / 4} \mathrm{~K}$,

and $\zeta \equiv 0.1 T_{\mathrm{b}, 8}-0.001 g_{14}^{1 / 4} \sqrt{0.7 T_{\mathrm{b}, 8}}$. Here $g_{14}$ is the surface gravity in units of $10^{14} \mathrm{~cm} \mathrm{~s}^{-2}, T_{\mathrm{b}, 8}$ is $T_{\mathrm{b}}$ in $10^{8} \mathrm{~K}$, and $T_{\mathrm{s}, 6}$ is $T_{\mathrm{s}}$ in $10^{6} \mathrm{~K}$.

The function $X$ has been fitted by decomposing into transversal and longitudinal parts as

$\mathcal{X}\left(B, \varphi, T_{\mathrm{b}}\right)=\left[\mathcal{X}_{\|}^{9 / 2}\left(B, T_{\mathrm{b}}\right) \cos ^{2} \varphi+\mathcal{X}_{\perp}^{9 / 2}\left(B, T_{\mathrm{b}}\right) \sin ^{2} \varphi\right]^{2 / 9}$,

where $\varphi$ is the angle between the MF and the normal to the surface. Potekhin \& Yakovlev (2001) give the following fits for $\mathcal{X}_{\perp}$ and $X_{\|}$

$$
\begin{aligned}
& \mathcal{X}_{\|}\left(B, T_{\mathrm{b}}\right)=1+0.0492 B_{12}^{0.292} T_{\mathrm{b}, 9}^{0.240} \\
& \mathcal{X}_{\perp}\left(B, T_{\mathrm{b}}\right)=\frac{\sqrt{1+0.1076 B_{12}\left(0.03+T_{\mathrm{b}, 9}\right)^{-0.559}}}{\left[1+0.819 B_{12} /\left(0.03+T_{\mathrm{b}, 9)}\right]^{0.6463}\right.}
\end{aligned}
$$

where $B_{12}=B$ in units of $10^{12} \mathrm{G}$. These fits are valid for $B<$ $10^{16} \mathrm{G}$ and $10^{7} \mathrm{~K} \leq T_{\mathrm{b}} \leq 10^{9.5} \mathrm{~K}$.

It must be recalled that the above $T_{\mathrm{b}}-T_{\mathrm{s}}$ relation is based on a plane-parallel approximation. When this approach is applied to a spherical star, meridional heat fluxes in the envelope are not allowed and, therefore, this approximation may be inaccurate when these fluxes compete with the purely radial ones. In addition, Chugunov \& Haensel (2007) find that the contribution of ions or phonons to the thermal conductivity of the envelope can reduce the anisotropy of heat conduction. Therefore, we have revisited the magnetized envelope problem with two motivations: upgrading the microphysical inputs (thermal conductivity) and assessing the accuracy of the plane-parallel approximation.

To avoid solving the hydrostatic equilibrium equations in two dimensions, we have built a spherically symmetric iron envelope model with a zero temperature equation of state. With this fixed background, we calculated stationary solutions of the heat transport equation in 2D, with a given MF geometry (dipole solution). At first glance, this may seem inaccurate, since finite temperature effects may be relevant at low density but, as explained in Gudmundsson et al. (1983), the main regulator of the $T_{\mathrm{b}}-T_{\mathrm{s}}$-relation is the sensitivity strip where the opacity is maximum. This strip marks the transition from electronic heat transfer to the radiation-dominated one. It lies at relatively high densities except for very low temperatures. Hence, structural changes at low density do not affect the solution, even for extreme cases such as a condensed surface (Potekhin et al. 2007). To test the validity of this assumption and to compare with previous works, we have taken the crustal MF to be either purely radial (labelled $\|$, parallel to the normal to the surface) or purely tangential to the surface (labelled $\perp$ ). In these two limiting cases, the heat flux is purely radial, and we have reproduced within a $5 \%$ the $T_{\mathrm{b}}-T_{\mathrm{s}}$ relation given by Potekhin \& Yakovlev (2001) if neutrino emissivities are not considered. The minor differences are probably due to the different EOS or NS model; i.e., our neutron drip point is at $\rho=3.5 \times 10^{11} \mathrm{~g} / \mathrm{cm}^{3}$, while theirs is at $\rho=4 \times 10^{11}$. Our results for purely parallel or purely tangential fields including the neutrino emissivity in the outer crust are compared to the analytical fit from Potekhin \& Yakovlev (2001)
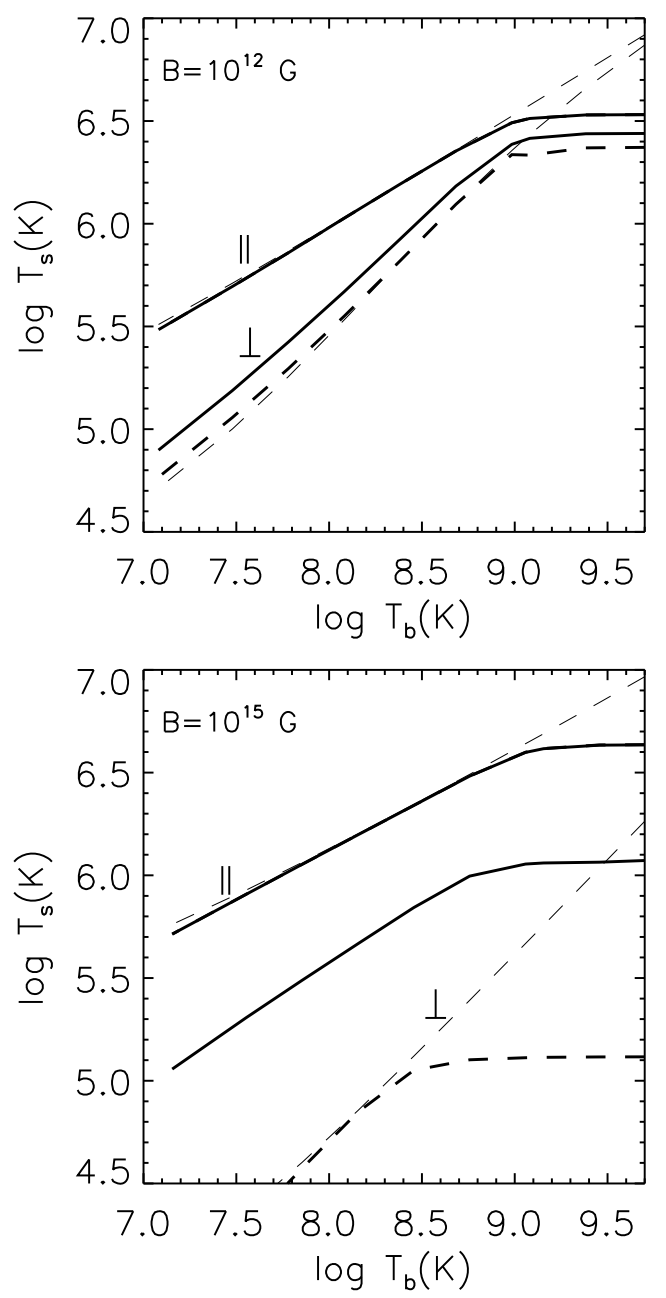

Fig. 1. Surface temperature as a function of temperature at the neutron drip point for two models of NS envelopes with different MF strengths. The thin dashed lines are the analytical fits from (Potekhin \& Yakovlev 2001) for MF parallel $(\|)$ and perpendicular $(\perp)$ to the normal direction to the surface. The thick dashes are our results for the $\perp$ case, and the solid lines are our full 2D results for a dipolar field (we show $T_{\mathrm{s}}$ at the pole and at the equator). Our results for a purely radial field cannot be distinguished from the upper solid line $\left(T_{\mathrm{s}}\right.$ at the pole for the dipolar $\mathrm{MF})$. Numerical results always include the effect of neutrino emission in the outer crust and envelope.

(without the effect of neutrino emissivity) in Fig. 1. The agreement is very good in those cases where neutrino emission is not important (low temperature). Moreover, our results for radial and tangential MF can be directly compared to Figs. 4 and 5 of Potekhin et al. (2007), in which neutrino emissivity effects are considered. The very good agreement with this work indicates that the use of a zero-temperature EOS for solving the hydrostatic equilibrium equation is a valid approximation. The same comment applies to the effects of strong MF in the low-density EOS, as long as they are only noticeable at densities where radiation dominates the heat transport. Finally, to check the effect of using an EOS in which the thermal contribution to the pressure is neglected to obtain the mechanical structure of the envelope, we also recalculated some of the models using new envelope structures obtained by solving the hydrostatic equilibrium equations for a finite temperature EOS and assuming the temperature profile of the previous models. We find the same surface temperatures in both models (within a $1 \%$ ), further confirming the validity of our approach. 
For 2D models, Fig. 1 shows our 2D transport results with a dipolar field. The quoted value of the MF corresponds to the strength at the magnetic pole. Our results confirm that the effect of ion/phonon transport in the envelope is to reduce the large anisotropy obtained in previous magnetized models. In addition, by performing $2 \mathrm{D}$ heat transport calculations through the magnetized envelope, we take into account the meridional heat fluxes driven by the meridional temperature gradients between pole and equator, and we find that the anisotropy is further reduced. This effect is more relevant for high fields, resulting in an equatorial temperature about a factor 3 lower than the polar temperature, in contrast to the 2-3 orders of magnitude obtained in previous models. For practical purposes, we made fits of our results keeping a similar functional form to Eq. (30), but only changing the form of $\mathcal{X}_{\|}$and $\mathcal{X}_{\perp}$ as follows:

$$
\begin{aligned}
& \mathcal{X}_{\|}\left(B, T_{\mathrm{b}}\right)=1+0.05 B_{12}^{0.25} T_{\mathrm{b}, 9}^{0.240} \\
& \mathcal{X}_{\perp}\left(B, T_{\mathrm{b}}\right)=\frac{\sqrt{1+0.07 B_{12}\left(0.03+T_{\mathrm{b}, 9}\right)^{-0.559}}}{\left[1+0.9 B_{12} /\left(0.03+T_{\mathrm{b}, 9}\right)\right]^{0.4}} .
\end{aligned}
$$

We find that this formula reproduces the correct numerical results for the $T_{\mathrm{b}}-T_{\mathrm{s}}$ relation very well for a dipolar field geometry. The ratio between polar and equatorial temperature is significantly lower than in the case of 1-D plane-parallel envelope models. We also confirm that, if neutrino emission at densities $\rho>10^{10} \mathrm{~g} / \mathrm{cm}^{3}$ is taken into account, there is an MF-dependent upper limit to the effective surface temperature (Potekhin et al. 2007), but our maximum temperatures for MF tangential to the surface are considerably higher than those obtained before. We find that the upper limit consistent with our numerical results is well-fitted by the following expressions

$$
\begin{aligned}
T_{\mathrm{s}, \|}^{\max } & =\frac{3.6 \times 10^{6} \mathrm{~K}}{1+0.02 \log B_{12}} \\
T_{\mathrm{s}, \perp}^{\max } & =\frac{2.8 \times 10^{6} \mathrm{~K}}{1+0.6 \log B_{12}} .
\end{aligned}
$$

It must be stressed that these upper limits are obtained by including the outer crust $\left(3 \times 10^{11}>\rho>10^{10} \mathrm{~g} / \mathrm{cm}^{3}\right)$ in the static envelope calculations that fix the inner envelope boundary condition. If the numerical grid of the cooling code extends to densities lower than $10^{10} \mathrm{~g} / \mathrm{cm}^{3}$, this effect is consistently incorporated and there is no need to include these corrections in the $T_{\mathrm{b}}-T_{\mathrm{s}}$ relation. We neglect the possibility of Joule heating within the envelope because, due to the very high magnetic diffusivity, initial electric currents are quickly dissipated and may only be important at the very beginning of a NSs life. Obviously these results may vary for other field geometries. We did check, however, that our improved fits (31) combined with Eq. (28) are a good approximation (within 5\%) to the numerical result in a number of cases. In Fig. 2 we compare the numerical results with the analytical fits for three different models. In the top panel the MF geometry is purely dipolar and the temperature distribution at the inner envelope is fixed to $T_{\mathrm{b}}=10^{7}\left(\cos ^{2} \theta+0.2 \sin ^{2} \theta\right) \mathrm{K}$. The middle panel shows the results for a model with the same MF configuration but a temperature distribution at the base of the envelope given by $T_{\mathrm{b}}=10^{7}\left(1+3 \sin ^{2} \theta\right) \mathrm{K}$. The bottom panel corresponds to a model with the same $T_{\mathrm{b}}$ distribution as the middle panel, but with a purely quadrupolar MF geometry. In all cases $B_{\mathrm{p}}=10^{15} \mathrm{G}$, so that the combination of high field and low temperature demonstrates the validity of the fit for the most extreme cases.
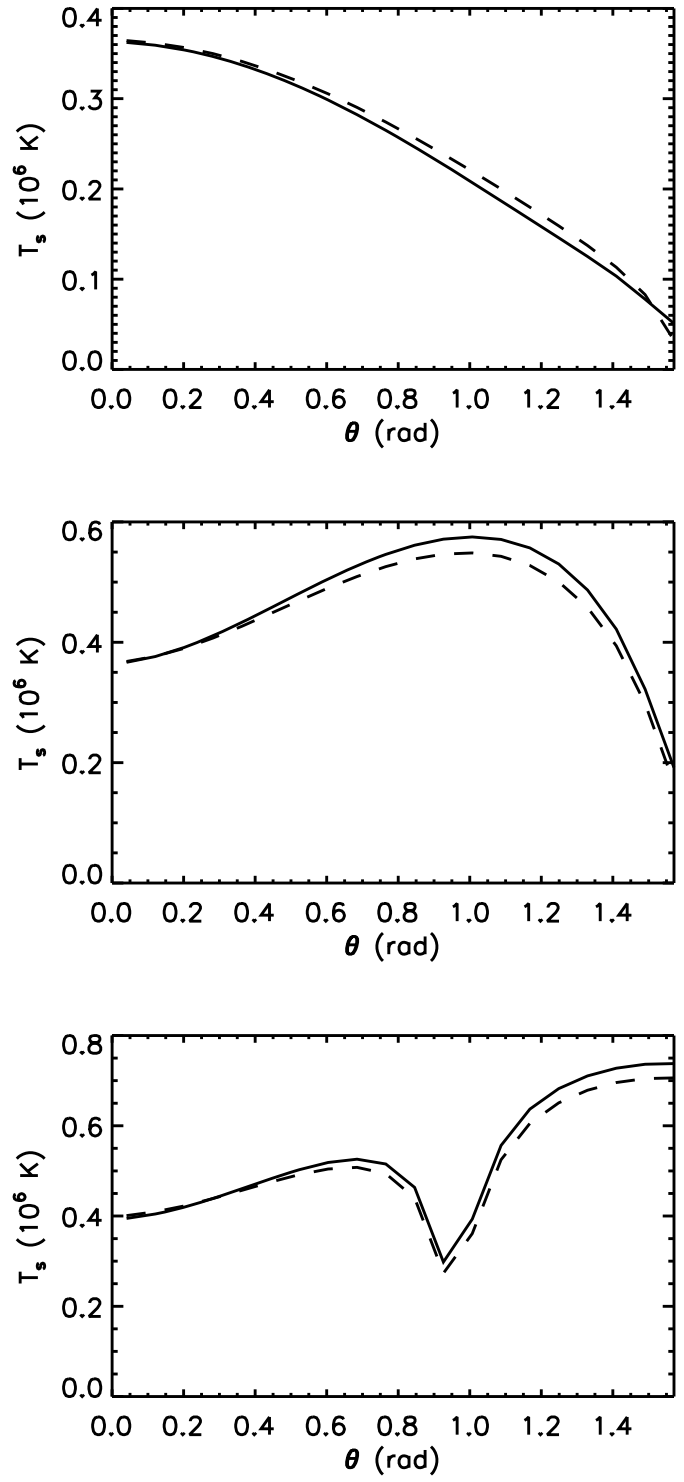

Fig. 2. Stationary 2D solutions of magnetized envelopes: surface temperature profiles as a function of the polar angle for $B_{\mathrm{p}}=10^{15} \mathrm{G}$ and three different combinations of the $T_{\mathrm{b}}$-distribution and MF geometry. The solid lines correspond to the numerical results and the dashed lines to the analytic fits from Eqs. (31) and (28).

\section{Results}

We present the results now of our numerical simulations of the magneto-thermal evolution of NSs and its dependence on the initial MF structure and strength. We restrict this presentation to a selection of the initial models described in Aguilera et al. (2008b). Our model A at $t=0$ consists of a dipolar $(n=1)$ poloidal field according to Eq. (13) of Aguilera et al. (2008b), parameterized by the value of the radial component at the magnetic pole, $B_{\mathrm{p}}$. In model B we add a quadrupolar $(n=2)$ toroidal component obeying Eq. (11) of Aguilera et al. (2008b) to the initial dipolar field, with a maximum value of $\approx 50 B_{\mathrm{p}}$. This model represents the case of a strong toroidal component confined in the crust. We recall that we focus on the purely diffusive case in this paper, i.e., the Hall term in the induction equation is neglected. This term needs a separate specific numerical treatment that is out of the current capabilities of our code and will be discussed in later work. Its influence can be important either at 


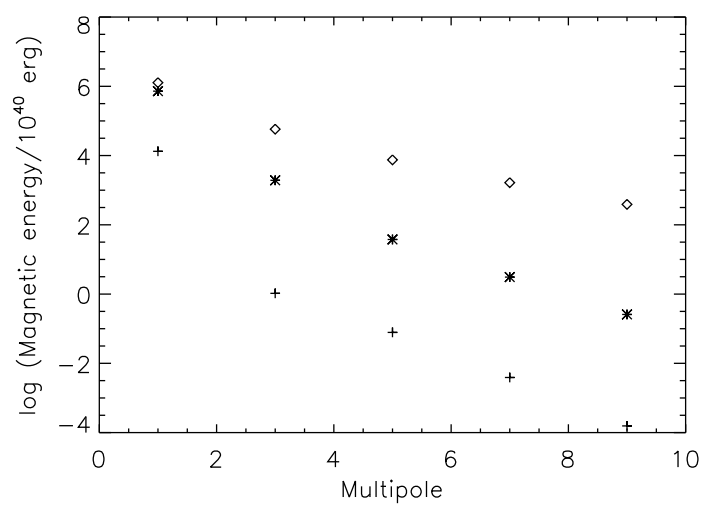

Fig. 3. Power spectrum at $t=5 \times 10^{5}$ years for the models corresponding to purely poloidal fields with initial values of $B_{\mathrm{p}}=10^{13}$ (crosses), $10^{14}$ (stars), and $10^{15} \mathrm{G}$ (diamonds).

very early times or, as we point out at the end of the section, at late times during the photon cooling era. For most of the first $10^{5}-10^{6}$ years of a NS life we do not expect qualitative changes from our present results.

To begin our discussion, we present in Fig. 3 the power spectra for model A with three initial fields of $B_{\mathrm{p}}=10^{13}, 10^{14}$, and $10^{15} \mathrm{G}$ at the age of $t=5 \times 10^{5}$ years. We see how the coupling between different modes due to the angular dependence of $\eta$ fills the shorter wavelength modes (initially only the dipolar poloidal component $n=1$ is present). Only odd multipoles are present because the initial model is symmetric with respect to the equatorial plane. At this age, the cascade has filled out all large wavenumber modes and is saturated following an $n^{-4}$ power law approximately for the cases with large initial fields. This result shows that, even if the influence of the nonlinear Hall term is negligible, the thermo-magnetic coupled evolution results in a complex field geometry that cannot be described by a single mode. Although the dominant mode is still the dipole, the distribution of part of the energy in higher order modes leads to a slightly faster dissipation.

In Fig. 4 we show the crustal temperature distribution of a NS at different ages $(t=10,100$ and $500 \mathrm{kyr})$, together with the poloidal field lines. The tendency of the surfaces of constant temperature to be aligned with the magnetic field lines discussed in Pérez-Azorín et al. (2006a) and Geppert et al. (2004) is clearly visible, but there is an important difference with respect to previous works. The consistent inclusion of the Joule heating source results in a larger energy deposition in the region where currents are more intense. In this particular model this happens at low latitudes, since the currents that maintain a poloidal field are toroidal. Thus more heat is released close to the equator. While the polar regions are always in thermal equilibrium with the core, the equator is insulated because of the reduced thermal conductivity across magnetic field lines. As a consequence, the heat released at early times in the equatorial region cannot flow across field lines into the core, where it would be rapidly lost by neutrino emission. It is only allowed to flow towards the surface along field lines. This modifies the traditionally accepted temperature distribution consisting of hot poles and a cooler equator (insulated from the warmer core). Instead we find that the equatorial region of the outer crust is actually warmer than the poles. In models with weak MFs, when the effect of Joule heating becomes less effective, the situation is inverted and the thermal distribution with hot polar caps is recovered. Notice the large difference between polar and equatorial temperatures, up to a factor of 2 at early times (the numbers next to the color scale indicate the maximum and minimum temperature at each evolutionary time), in contrast with the nearly isothermal crusts at late times.

In non magnetized NSs the heat conduction between the crust and the core is able to counteract the local neutrino emission processes and produce an isothermal NS after the thermal relaxation stage, which lasts 10-100 years. This interchange of energy between core and crust is still important in magnetized neutron stars, but the strong tangential field in the equatorial region of the inner crust has the effect of increasing the thermal relaxation time in several orders of magnitude, so that the equatorial region in Fig. 4 effectively becomes decoupled from the core.

We must recall that the anisotropy in $T_{\mathrm{b}}$ (at the bottom of the envelope) is not necessarily the same as that of $T_{\mathrm{s}}$. The blanketing effect of the envelope, and/or atmospheric effects should be taken into account before a comparison with observations. In Fig. 5 we plot the evolution of both the temperature at the bottom of the envelope (top panel) and the surface temperature (bottom panel) as a function of the polar angle.

In Fig. 6, we show a sample of cooling curves (effective temperature versus true age) for models A and B but varying the initial strength of the field. For high fields the effect of Joule heating is visible from the very beginning of the evolution. The effective temperature of a young, $t=10^{3} \mathrm{yr}$ magnetar with $B_{\mathrm{p}}=10^{15} \mathrm{G}$ is higher by a factor of 2 than that of a NS with a standard $B_{\mathrm{p}}=10^{13} \mathrm{G}$, and it is kept nearly constant for a much longer time. The effect is further enhanced in model B thanks to the extra energy stored in the toroidal field. In spite of some quantitative differences, our improved simulations confirm the qualitative results described in Aguilera et al. (2008c), where a phenomenological MF decay law has been adopted. The most important difference is the somewhat higher temperature reached in these models, when compared to Aguilera et al. (2008c). The difference stems from the particular location of the heat dissipated by the Joule effect in the regions where currents are intense, as opposed to the homogeneous distribution of energy in the formerly adopted phenomenological model.

In Fig. 7 we show the value of the MF at the pole as a function of time for models A and B with different initial field strengths. Naively, we can divide the models in two groups: strong initial field $B>5 \times 10^{13} \mathrm{G}$ and weak initial field $B<5 \times 10^{13} \mathrm{G}$. The plot shows that models with strong initial fields are subject to a faster decay than those with weaker fields. Models with weak initial fields are subject to decay in a more or less similar manner. The field typically decays in about a factor of two on a timescale of few $10^{6}$ years and then remains nearly constant, due to the increase in the magnetic diffusion timescale as the star cools down.

Models with initially large fields behave in a different way. Since the magnetic energy stored in the crust is now large enough to significantly affect the thermal evolution when it is steadily released by Joule heating, it results in a higher average temperature of the crust. But this process has a back-reaction: the higher temperatures imply higher resistivity and therefore faster decay. Interestingly, at $t>10^{6} \mathrm{yr}$, all models with large poloidal initial fields seem to converge to an asymptotic fiducial value of $B_{\text {asymp }} \approx 4 \times 10^{13} \mathrm{G}$, while all models with strong toroidal fields converge to a lower value of $B_{\text {asymp }} \approx 1.5 \times 10^{13} \mathrm{G}$, because of the higher temperatures reached on average during the evolution.

Based on these results we predict that all sufficiently old NSs born with $B_{\mathrm{p}}$ above a critical value evolve towards similar field strengths, while those born with lower fields show a 

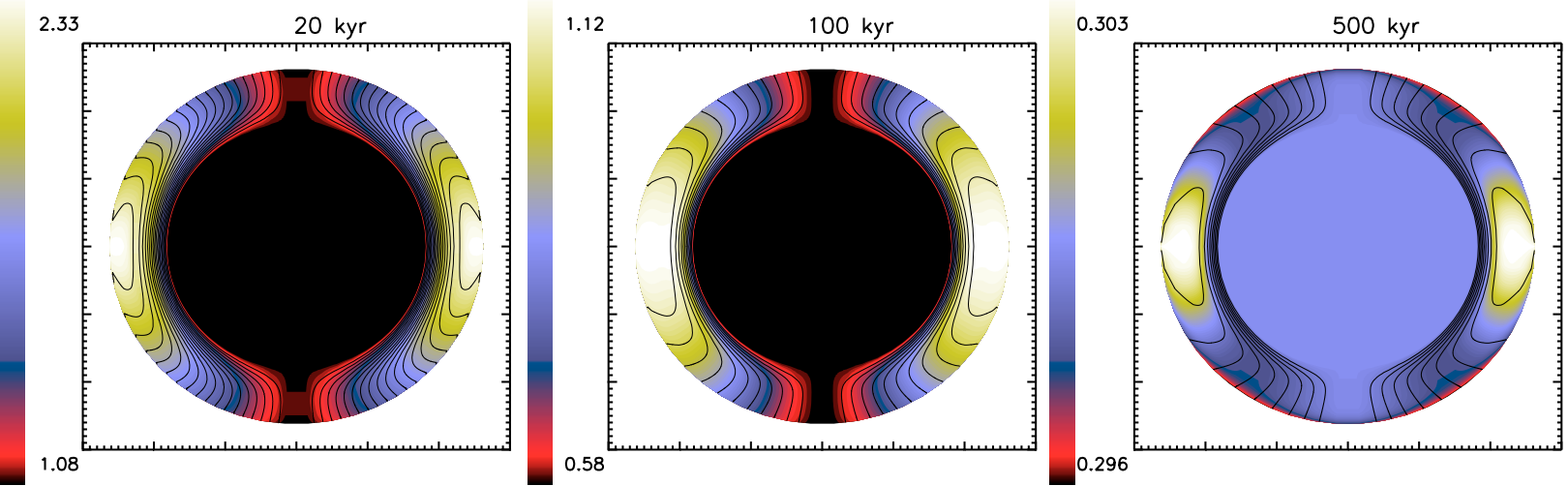

Fig. 4. Temperature distribution in the crust of a NS (i.e. up to the bottom of the envelope) at different ages. The initial MF is purely poloidal with $\left(B_{\mathrm{p}}=10^{14} \mathrm{G}\right)$. The field lines are also shown. The numbers on the color-scale on the left of each figure indicate the maximum and minimum values of the temperature (in units of $10^{8} \mathrm{~K}$ ) at each age. The crustal shell has been stretched a factor of 4 for clarity.
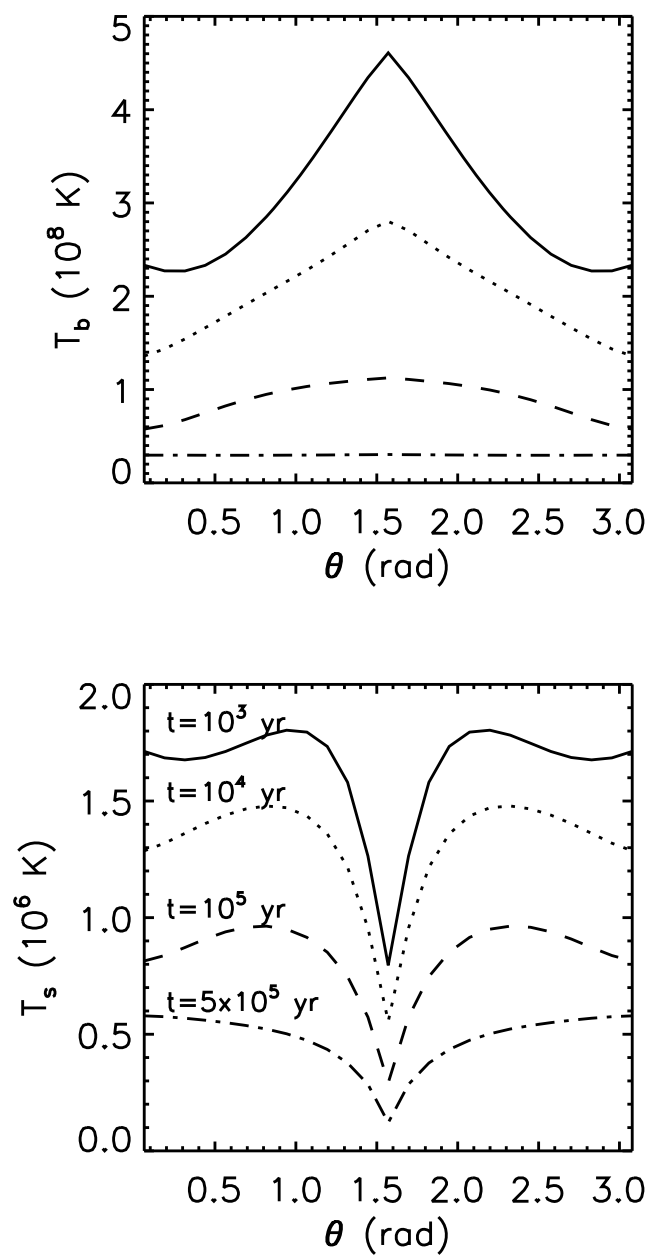

Fig. 5. Temperature profiles at the base of the envelope $\left(T_{\mathrm{b}}\right)$ and at the surface $\left(T_{\mathrm{s}}\right)$ at different evolutionary times. The initial MF is purely poloidal with $B_{\mathrm{p}}=10^{14} \mathrm{G}$.

MF distribution at late times similar to the initial distribution but shifted in about a factor of 2 to lower values. This critical initial field is approximately $B_{\text {crit }} \approx 5 \times 10^{13} \mathrm{G}$. It also delimits the minimum field strength that can actually influence the thermal evolution of the NS by MF dissipation. Once the MF is dissipated below that value, its influence on the later evolution is reduced and the subsequent evolution proceeds in a similar

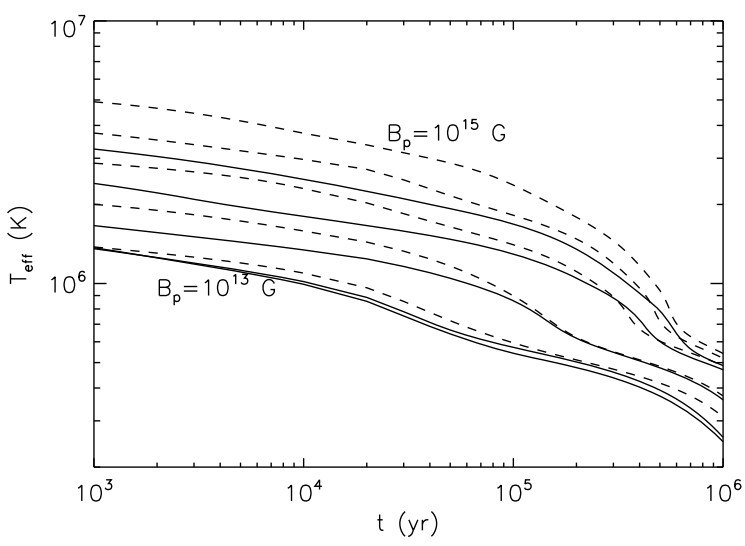

Fig. 6. Cooling curves. Effective temperature as a function of age for different initial field strengths (from bottom to top $B_{\mathrm{p}}=10^{13}, 3 \times$ $10^{13}, 10^{14}, 3 \times 10^{14}$, and $\left.10^{15} \mathrm{G}\right)$. The solid lines correspond to Model A and the dashed lines to model B.

way in all cases. Our models predict that no old magnetar can be found, and that, at ages $t>5 \times 10^{5} \mathrm{yr}$, the typical MF for all NSs born as magnetars must be $\operatorname{similar}\left(B \approx B_{\text {asymp }}\right)$. Up to now, observational data do not contradict this fact.

To conclude this section we turn now to discussing the evolution of the dimensionless magnetization parameter $\omega_{B} \tau$, where $\omega_{B}$ is the electron gyro-frequency and $\tau$ is the electron relaxation time. The Hall induction Eq. (6) can also be written as

$$
\frac{\partial \boldsymbol{B}}{\partial t}=-\frac{c^{2}}{4 \pi} \boldsymbol{\nabla} \times\left(\frac{1}{\sigma_{\|}}\left\{\boldsymbol{\nabla} \times\left(\mathrm{e}^{v} \boldsymbol{B}\right)+\omega_{B} \tau\left[\boldsymbol{\nabla} \times\left(\mathrm{e}^{v} \boldsymbol{B}\right)\right] \times \boldsymbol{e}_{\boldsymbol{B}}\right\}\right),
$$

where $\boldsymbol{e}_{\boldsymbol{B}}$ is the unit vector in the direction of $B$.

In this form, the interpretation of $\omega_{B} \tau$ is straightforward: when the magnetization parameter exceeds unity, the Hall drift term dominates. The effect of the Hall drift term at early times has been discussed in detail in (Pons \& Geppert 2007). As a rule of thumb, when $\omega_{B} \tau>10^{3}$, the effect of the Hall drift significantly changes the evolution, while more moderate values lead to a somewhat faster dissipation due to reorganization of the field in smaller scales. Because of numerical limitations, in this work we have restricted ourselves to the purely diffusive case, but it is worth looking at the evolution of this parameter as shown in Fig. 8. Here we show radial profiles of the magnetization parameter in the crust for model $\mathrm{A}$ and three different initial values of $B_{\mathrm{p}}$. The upper panel corresponds to $t=10^{4} \mathrm{yr}$ and the lower 


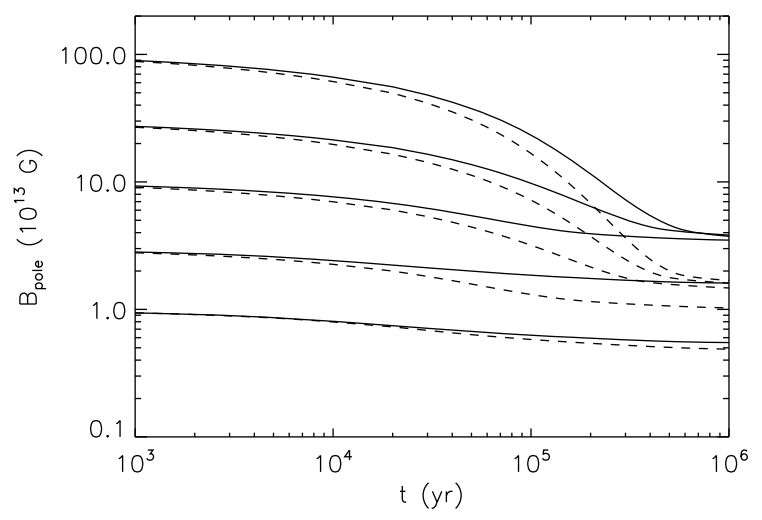

Fig. 7. Evolution of the MF strength at the pole $B_{\mathrm{p}}$ during the first million years of a NS for several initial values. The solid lines correspond to Model A and the dashed lines to model B.

panel to $t=10^{6}$ years. At early times, the scaling of $\omega_{B} \tau$ with $B_{\mathrm{p}}$ is visible and only in magnetars must one expect high values in the inner crust. However, the back-reaction of the field evolution on the temperature has some interesting implications. At late times, the temperature of low-field NSs is lower than that of highly magnetized NS, so that the temperature dependence of the electron relaxation time overcomes the effect of the MF, and it turns out that the former magnetars are less magnetized than NSs born with moderate fields. In addition, at ages of $\approx 10^{6} \mathrm{yrs}$, the temperature is low enough to ensure that the magnetization parameter is very large for all models studied, especially in the inner crust (where the conductivity is higher). This opens up more questions about the late evolution of NSs that is likely to be dominated by the nonlinear Hall term in most NSs. In particular, the possibility of the Hall instability (Rheinhardt \& Geppert 2002) or implications on the evolution of the braking index are worth exploring.

\section{Conclusions}

We performed consistent 2D simulations of the coupled magneto-thermal evolution of NSs for the first time, including realistic microphysical input and general relativistic corrections. By properly taking the interplay of the MF and temperature evolution into account in cooling simulations, we have found that their mutual influence is important and affects the outcome when the initial field strength is close to or larger than a critical value of $B_{\text {crit }}=5 \times 10^{13} \mathrm{G}$. It has effects on both the temperature and the field strength.

The average effective temperature of a NS born with $B_{\mathrm{p}}<$ $B_{\text {crit }}$ is barely affected, while those born as magnetars are subject to significant heating by the dissipation of currents in the crust. In addition, since heating is locally important in the regions where currents are more intense, the surface temperature distribution can be very different depending on the field geometry. While the pole (or other regions in which the field lines are nearly radial through the crust) is in thermal equilibrium with the core and has its same temperature, regions in which the field lines are tangential remain essentially thermally isolated. This can produce cooler areas if no heating process is considered or, conversely, hotter regions if heating is important. This is the case of poloidal fields, in which currents are located near the equatorial region which remains warmer than the rest of the crust during a long time. The effective temperature of models with strong internal toroidal components are systematically higher than that
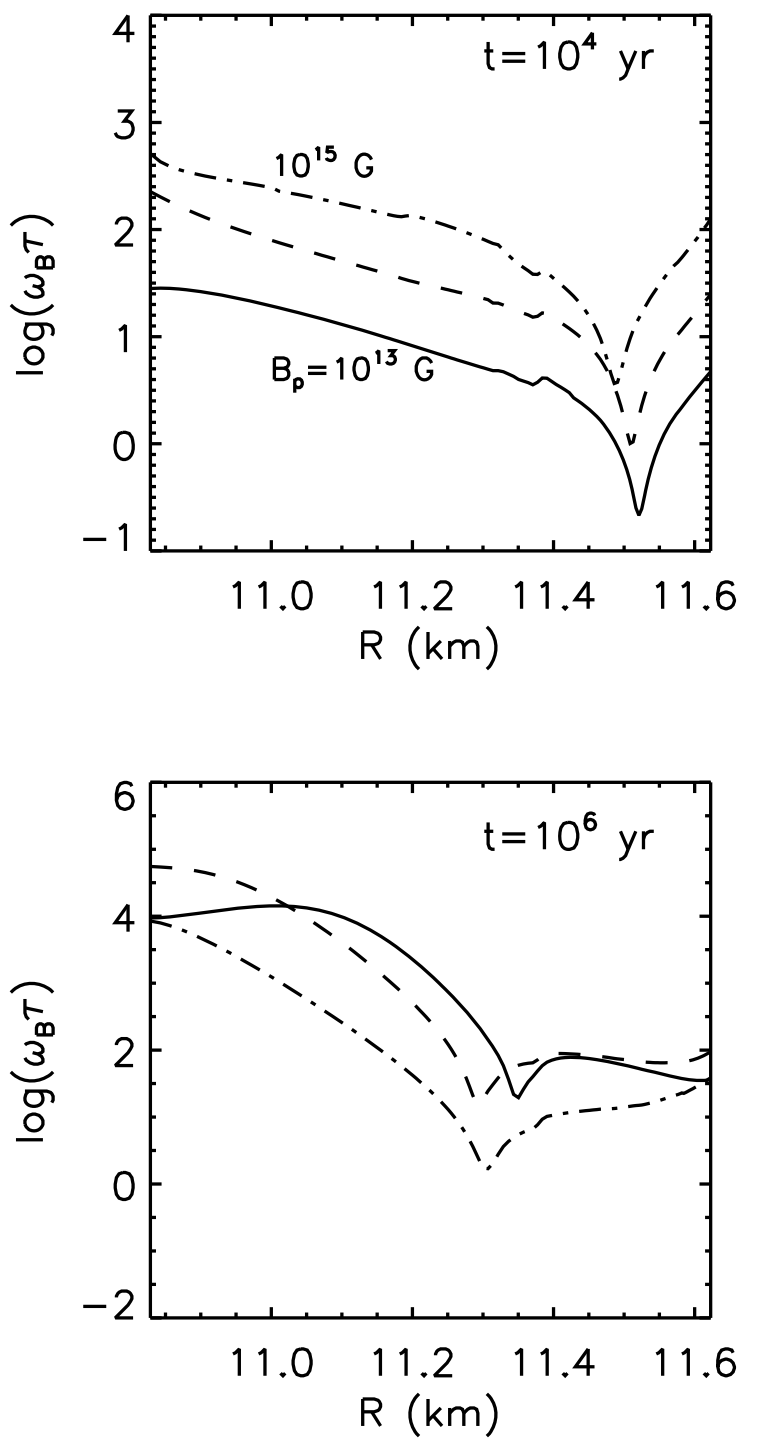

Fig. 8. Radial equatorial profiles of the magnetization parameter $\omega_{B} \tau$ for model $A$ and three different initial values of $B_{\mathrm{p}}: 10^{13} \mathrm{G}$ (solid lines), $10^{14} \mathrm{G}$ (dashed lines), and $10^{15} \mathrm{G}$ (dotted lines). The upper panel corresponds to $t=10^{4} \mathrm{yr}$ and the lower panel to $t=10^{6}$ years.

of models with purely poloidal fields, due to the additional energy reservoir stored in the toroidal field that is gradually released as the field dissipates.

In the models with stronger fields, and as a result of the average higher temperatures, the crustal electrical resistivity is enhanced and magnetic diffusion proceeds faster during the first $10^{5}-10^{6}$ years of a NS's life. As a consequence, all NSs born with fields larger than a critical value $\left(>5 \times 10^{13} \mathrm{G}\right)$ reach similar field strengths $\left(\approx 2-3 \times 10^{13} \mathrm{G}\right)$ at late times, irrespective of the initial strength. After $10^{6}$ years the temperature is so low that the magnetic diffusion timescale becomes longer than the typical ages of radiopulsars, resulting in apparently no dissipation of the field in old NSs. We confirm the strong correlation between the MF and the surface temperature of relatively young NSs discussed in preliminary works. If the MF of magnetars is caused by superconducting currents in the core (as opposed to crustal currents), the longer diffusion timescale in the core would allow magnetars to live much longer with their original large fields. 
Thus, observations of magnetars can help to discern between models with currents located in the crust or in the core.

It should be mentioned that magnetic field evolution by ambipolar diffusion in the core may produce qualitatively similar effects to those obtained in this work, keeping the temperature high while the field is strong and stopping field decay when the temperature drops (see e.g. Reisenegger 2008). In this work we have not considered the MF evolution in the core because ambipolar diffusion is usually considered under the assumption that the core is in a nonsuperfluid state and therefore is important during the very early stages of evolution. We are more interested in the longterm evolution, after the temperature rapidly drops below the critical temperature for nucleon superfluidity. To quantify the relative importance of both effects one would need to consider ambipolar diffusion in a superconducting fluid coupled to the dissipation of crustal currents studied here.

The detection of magnetars with true ages (the spin-down age can be seriously overestimated) $t \approx 10^{6} \mathrm{yr}$, or the detection of a young highly magnetized NS with $T<10^{6} \mathrm{~K}$ would be a serious challenge for crustal field models. At present our results agree with the known population of high field NSs and magnetars and support the idea of the existence of a strong crustal MF component in magnetars.

Given the complexity of the feedback between temperature and MF, it seems necessary to extend this work in two main lines that can shed new light on our knowledge of the cooling theory of NS: engaging 3D simulations and including the Hall term in the induction equation. The complex geometry that may arise in a realistic case with hot spots and irregular fields is certainly not treatable with our present code and needs further investigation. Similarly, the influence of the Hall term at late times when the temperature is so low that the magnetic diffusivity is negligible or a consistent treatment of ambipolar diffusion in a superconducting fluid coupled to the dissipation of crustal currents can produce new interesting effects so both are issues worth exploring in future work.
Acknowledgements. We thank D.N. Aguilera for valuable comments and providing updated conductivity routines used in the simulations, and Andreas Reisenegger for a critical reading and his constructive and helpful comments. This research has been supported by the Spanish MEC grant AYA 2007-67626C03-02 and the Research Network Program Compstar funded by the ESF. U. Geppert thanks the University of Alicante for support under its visitors program.

\section{References}

Aguilera, D. N., Cirigliano, V., Pons, J. A., Reddy, S., \& Sharma, R. 2008a, ArXiv e-prints, 807

Aguilera, D. N., Pons, J. A., \& Miralles, J. A. 2008b, A\&A, 486, 255

Aguilera, D. N., Pons, J. A., \& Miralles, J. A. 2008c, ApJ, 673, L167

Canuto, V., \& Chiuderi, C. 1970, Phys. Rev. D, 1, 2219

Chugunov, A. I., \& Haensel, P. 2007, MNRAS, 381, 1143

Flowers, E., \& Itoh, N. 1976, ApJ, 206, 218

Geppert, U., Küker, M., \& Page, D. 2004, A\&A, 426, 267

Geppert, U., Küker, M., \& Page, D. 2006, A\&A, 457, 937

Gudmundsson, E. H., Pethick, C. J., \& Epstein, R. I. 1983, ApJ, 272, 286

Hoyos, J., Reisenegger, A., \& Valdivia, J. A. 2008, A\&A, 487, 789

Itoh, N. 1975, MNRAS, 173, 1P

Jones, P. B. 1999, Phys. Rev. Lett., 83, 3589

Landau, L., \& Lifshitz, E. 1960, Electrodynamics of Continuous Media (Addison Wesley)

Miralles, J. A., Urpin, V., \& Konenkov, D. 1998, ApJ, 503, 368

Page, D., Geppert, U., \& Zannias, T. 2000, A\&A, 360, 1052

Page, D., Lattimer, J. M., Prakash, M., \& Steiner, A. W. 2004, ApJS, 155, 623

Pérez-Azorín, J. F., Miralles, J. A., \& Pons, J. A. 2006a, A\&A, 451, 1009

Pérez-Azorín, J. F., Pons, J. A., Miralles, J. A., \& Miniutti, G. 2006b, A\&A, 459, 175

Pons, J. A., \& Geppert, U. 2007, A\&A, 470, 303

Pons, J. A., Link, B., Miralles, J. A., \& Geppert, U. 2007, Phys. Rev. Lett., 98, 071101

Potekhin, A. Y., \& Yakovlev, D. G. 2001, A\&A, 374, 213

Potekhin, A. Y., Chabrier, G., \& Yakovlev, D. G. 2007, Ap\&SS, 308, 353

Rädler, K.-H., Fuchs, H., Geppert, U., Rheinhardt, M., \& Zannias, T. 2001, Phys. Rev. D, 64, 083008

Reisenegger, A. 2008, ArXiv e-prints, 809

Rheinhardt, M., \& Geppert, U. 2002, Phys. Rev. Lett., 88, 101103

Urpin, V., \& Konenkov, D. 2008, A\&A, 483, 223

Ziman, J. 1979, Principles of the Theory of Solids (Cambridge University Press) 